\title{
ON AN INEQUALITY OF LYAPUNOV
}

\section{HARRY HOCHSTADT ${ }^{1}$}

Nehari [1] claims that if an interval $[a, b]$ contains $n$ zeros of a nontrivial solution of

$$
y^{(n)}+p_{n} y^{(n-1)}+\cdots+p_{1} y=0
$$

then

$$
\sum_{k=1}^{n} 2^{k}(b-a)^{n-k} \int_{a}^{b}\left|p_{k}\right| d t \geqq 2^{n+1} .
$$

According to Fink and St. Mary [2] the proof of (2) given in [1] is incorrect, and therefore the inequality is, as yet, undecided. For $n=2$ and $p_{2} \equiv 0,(2)$ is known to be correct and also the best possible. This case was first proved by Lyapunov and is generally referred to as Lyapunov's theorem. A recent proof of this result may be found in Hochstadt [3]. In [2] a similar technique is used to prove (2) for $n=2$, where $p_{2}$ is merely integrable on $[a, b]$. In fact, one can extract from [2] the inequality

$$
\left[(b-a) \int_{a}^{b}\left|p_{1}\right| d t\right]^{1 / 2}+\frac{1}{2} \int_{a}^{b}\left|p_{2}\right| d t \geqq 2 .
$$

The purpose of this note is to provide a generalization of (3) to certain $n$th order differential equations.

THEOREM. Consider the differential equation

$$
y^{(n)}-p y^{(n-1)}-q y=0, \quad n \geqq 2,
$$

where $p$ and $q$ are integrable on $[a, b]$. Suppose that a nontrivial solution of (4) has at least $n$ zeros on $[a, b]$. Then

$$
\left[(b-a)^{n-1} \int_{a}^{b}|q| d t\right]^{1 / n}+\frac{1}{n} \int_{a}^{b}|p| d t \geqq 2 .
$$

Clearly for $n=2,(5)$ reduces to (3). In order to prove (5) we reduce (4) to a system, by letting

$$
x_{i}=y^{(i-1)}, \quad i=1,2, \cdots, n,
$$

so that

Received by the editors October 16, 1968.

1 The author gratefully acknowledges the support of the National Science Foundation under Grant GP-8754. 


$$
\begin{aligned}
x_{i}^{\prime} & =x_{i+1}, \quad i=1,2, \cdots, n-1, \\
x_{n}^{\prime} & =p x_{n}+q x_{1} .
\end{aligned}
$$

Since $y$ vanishes $n$ times on $[a, b]$, each $x_{i}$ vanishes at least once on that interval. We can, therefore, split $[a, b]$ into two subintervals $[a, c]$ and $[c, b]$ where $a<c<b$ such that on each, each $x_{i}$ vanishes at least once.

First we shall consider the interval $[a, c]$, and let $\bar{x}_{i}$ denote the maximum of $\left|x_{i}\right|$ on that interval. Using (6) and the fact that each $x_{i}$ vanishes at some point on $[a, c]$ we have

$$
\begin{gathered}
\bar{x}_{i} \leqq \bar{x}_{i+1}(c-a), \quad i=1,2, \cdots, n-1, \\
\left|x_{n}\right| \leqq \bar{x}_{1} \int_{a}^{c}|q| d t+\int_{t}^{c}|p|\left|x_{n}\right| d t,
\end{gathered}
$$

where $x_{n}(c)=0$. From $(7)$ we see that

$$
\bar{x}_{1} \leqq \bar{x}_{n}(c-a)^{n-1}
$$

and combined with (8) we finally have

$$
\left|x_{n}\right| \leqq \bar{x}_{n}(c-a)^{n-1} \int_{a}^{c}|q| d t+\int_{t}^{c}|p|\left|x_{n}\right| d t .
$$

From (9) by means of Gronwall's inequality $[4$, p. 24$]$ we find that

$$
\bar{x}_{n} \leqq \bar{x}_{n}(c-a)^{n-1} \int_{a}^{c}|q| d t \exp \int_{a}^{c}|p| d t
$$

and finally

$$
\int_{a}^{c}|q| d t \geqq \frac{\exp \int_{a}^{c}|p| d t}{(c-a)^{n-1}}
$$

Similarly

$$
\int_{c}^{b}|q| d t \geqq \frac{\exp \int_{c}^{b}|p| d t}{(b-c)^{n-1}} .
$$

We can combine (10) and (11) and use the inequality

$$
A^{n} / a^{n-1}+B^{n} / b^{n-1} \geqq(A+B)^{n} /(a+b)^{n-1}
$$

to obtain 


$$
\begin{aligned}
(b-a)^{n-1} \int_{a}^{b}|q| d t \geqq & {\left[\exp \left(-\frac{1}{n} \int_{a}^{c}|p| d t\right)\right.} \\
& \left.+\exp \left(-\frac{1}{n} \int_{c}^{b}|p| d t\right)\right]^{n}
\end{aligned}
$$

(12) is an interesting inequality in its own right and for $n=2$ is also stated in [2]. In order to derive the simpler and desired inequality (5) we use the fact that

$$
\exp (-x) \geqq 1-x
$$

in (12) and extract the $n$th root of both sides. Then

$$
\begin{aligned}
{\left[(b-a)^{n-1} \int_{a}^{b}|q| d t\right]^{1 / n} } & \geqq 2-\frac{1}{n}\left(\int_{a}^{c}|p| d t+\int_{c}^{b}|p| d t\right) \\
& =2-\frac{1}{n} \int_{a}^{b}|p| d t
\end{aligned}
$$

which is equivalent to (5).

\section{BibLIOGRAPHY}

1. Z. Nehari, On an inequality of Lyapunov, Studies in Mathematical Analysis and Related Topics, Stanford Univ. Press, Stanford, Calif., 1962, pp. 256-261.

2. A. M. Fink and D. F. St. Mary, On an inequality of Nehari, Proc. Amer. Math. Soc. 21 (1969), 640-642.

3. H. Hochstadt, $A$ new proof of a stability estimate of Lyapunov, Proc. Amer. Math. Soc. 14 (1963), 525-526.

4. P. Hartman, Ordinary differential equations, Wiley, New York, 1964.

PolyteChNic INSTITUTE OF BROOKL yN 